\title{
Web-based User Interface for Electrical Cell-substrate Impedance Sensing System
}

\author{
Chan-Young Park, ${ }^{1,2}$ Mi-So Lee, ${ }^{1,2}$ Yu-Seop Kim, ${ }^{1,2}$ \\ Hye-Jeong Song, ${ }^{1,2}$ and Jong-Dae Kim ${ }^{1,2 *}$
}

\footnotetext{
${ }^{1}$ Department of Convergence, Hallym University, 1 Hallymdaehak-gil, Chuncheon-si, Gangwon-do 24252, Korea

${ }^{2}$ Bio-IT Research Center, Hallym University, 1 Hallymdaehak-gil, Chuncheon-si, Gangwon-do 24252, Korea
}

(Received November 30, 2017; accepted April 16, 2018)

Keywords: electrical cell-substrate impedance sensing, web-based graphical user interface

In this paper, we propose a compact electrical cell-substrate impedance sensing (ECIS) system and a user interface for control and monitoring over the Internet. Since the proposed system is much smaller than existing devices and does not need input/output (I/O) devices, it is easy to install and prevents inadvertent user interaction. The proposed system can increase the efficiency of biological testing devices such as ECIS systems that require long-term experiments at long intervals. The temporal impedance change is measured and recorded on the embedded computer in the proposed system, and the resulting data can be displayed and controlled over the Internet using a web-based graphical user interface (GUI). Bioengineers are currently using the proposed systems for cell culture experiments and are very satisfied with the ease of installation and the ability to observe the experimental situation anytime and anywhere.

\section{Introduction}

Electrical cell-substrate impedance sensing (ECIS) is an electrical biosensing method for the micromotion measurement, drug response analysis, and barrier function assessment of cancer or stem cells. ${ }^{(1-6)}$ ECIS is based on electrical impedance spectroscopy, which uses the phenomenon that impedance increases between electrodes as cell mobility increases. ${ }^{(7)}$ Since the results from ECIS are interpreted by analyzing the changes in impedance over time, the graphical display of this change is essential. Commonly, a display device such as a liquidcrystal display (LCD) is employed in the system to provide a graphical user interface (GUI). However, since the impedance signal is measured for several days with intervals of several minutes (typically $5 \mathrm{~min}$ in a cell culture experiment), ${ }^{(1)}$ the user does not need continuous observation. When a display device such as an LCD is mounted on the same system with flexible user input devices such as a mouse and a keyboard, the overall size of the device will increase, as well as the possibility of careless user interactions. The aforementioned issue becomes more significant if such an ECIS system is implemented in the form of a point-ofcare-test system. Moreover, the next wave of computing era is beyond the reach of traditional

*Corresponding author: e-mail: kimjd@hallym.ac.kr https://doi.org/10.18494/SAM.2018.1818 
desktops. Many of the sensing systems surrounding us in the cyber-physical system (CPS) paradigm will be invisibly embedded in the environment and present in a form on the network. ${ }^{(8-10)}$ With this trend, the ECIS system will also be integrated into the environment and connected to the Internet. It is therefore desirable to reduce the size of the system and to be able to remotely control and monitor the system over the Internet. The data generated by the ECIS system will ultimately be linked and analyzed in the cloud database. Accordingly, the visualization of data or analysis results will also be performed on smart devices connected to the Internet. When the sensing system is developed in CPS format, it is important to analyze and design the user interface, which is very different from the existing desktop environment. ${ }^{(11)}$

As the popularity of GUI increased, the number of biomedical instruments equipped with a GUI to provide an active user interaction also increased in the past few decades. The use of a PC or its embedded versions equipped with Windows is now commonly seen in the implementation of these GUI functions, given its advantages such as a comfortable development environment and excellent maintenance. ${ }^{(11-13)}$ Recently, with its rapid development, open hardware and software platforms have been expected to be actively applied to provide a GUI. ${ }^{(14-16)}$ Regardless of the platform, the GUI has been implemented as a desktop application. However, owing to the development of Internet-of-Things and cloud computing, the display and data recording of biomedical equipment are expected to be connected to the cloud for maintenance, control, and monitoring. Following these changes, the implementation of the GUI on the web is also anticipated. ${ }^{(10,14,17)}$ Implementing the GUI of biomedical devices on the web will prevent inadvertent input by removing easy input devices such as a keyboard or a mouse. In addition, the space required for the input/output (I/O) device will be further reduced, providing a more spacious environment for the user.

In this paper, we propose a compact CPS-type ECIS system using the latest USB oscilloscope and mini PC, which is continuously developed. We also proposed a web-based user interface suitable for the user interface of this type of system so that control and monitoring can be performed via the Internet. The proposed system is currently being used in cell culture experiments and bioengineers are very happy to be able to monitor and discuss the experimental situation alone or with colleagues anytime and anywhere.

In the next section, the whole system and software structure are described to accomplish the purpose of this study, and the implementation results and conclusions are shown in Sects. 3 and 4, respectively.

\section{Implementation}

Figure 1 shows the block diagram of the ECIS system presented in this paper, which can monitor the impedance of 8 electrode channels simultaneously. An embedded computer controls the analog-front-end (AFE) block to periodically measure the impedance of 8 electrode channels and store them in a local storage unit or database. The web server implemented in the embedded computer receives the user request from the web front end and controls the AFE or displays the measured results. The AFE has a 2-channel signal acquisition system and a signal generator to measure the impedance as shown in Fig. 2. A sine wave with a peak-to- 


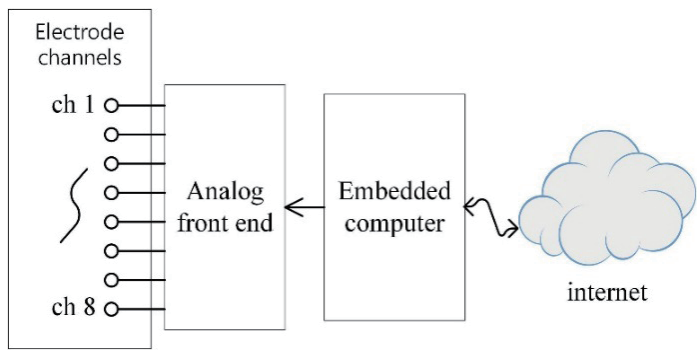

Fig. 1. Block diagram of ECIS system with webbased user interface.

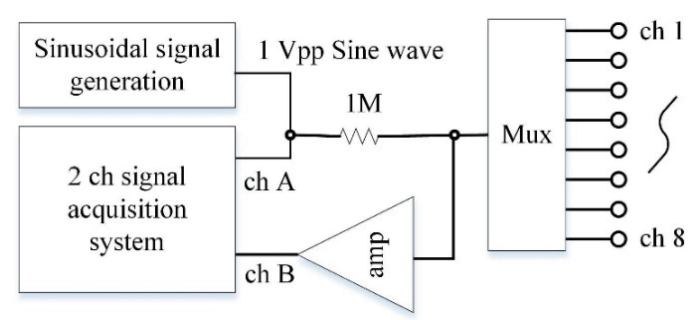

Fig. 2. AFE of the proposed ECIS system.

peak of $1 \mathrm{~V}$ is generated from the signal generator to measure the voltage across the channel by connecting $1 \mathrm{M} \Omega$ in series with the channel. The 2-channel signal acquisition system acquires the excitation and divided voltage signals concurrently.

From the various methods used to obtain the impedance from the signals, the fitting method with the least-squares error method was selected to estimate the sine functions of the excitation and divided voltage signals in this study.

The complex gain between the excitation and divided voltage signals can be calculated from the amplitude ratio and the phase difference between the fitted sine functions. Then, the impedance relative to the reference resistance ( $1 \mathrm{M} \Omega$ resistor in Fig. 2) is related to the calculated gain as

$$
\boldsymbol{g}=\frac{\boldsymbol{Z}}{\boldsymbol{Z}_{\text {ref }}+\boldsymbol{Z}},
$$

where $\boldsymbol{g}, \boldsymbol{Z}$, and $\boldsymbol{Z}_{\text {ref }}$ are the complex gain, the electrode impedance to be measured, and the reference resistance $(1 \mathrm{M} \Omega)$, respectively. From the above equation, the impedance of the electrode can be estimated.

A commercial USB oscilloscope (Analog Discovery 2, Digilent Inc., USA) had a signal generator along with a 2-channel signal acquisition system. It also provides an output port for the amplifier bias and analog mux switching.

The AFE is connected to an embedded computer via USB and receives power and data as shown in Fig. 3. Because the AFE section must be in the incubator, the USB cable needs to be extracted from the incubator for user interaction. Therefore, a flat cable was selected for the USB connection to enable the tight sealing of the incubator. A mini PC (Voyo mini, Shenzhen Voyo Technologies Co., Ltd., China) was employed for the embedded computer and connected to the Internet via Ethernet.

The embedded computer must be able to start the measurement and recording according to the user's request, to pause recording when the user wants to observe the status of the cell in the channel using the microscope, and then to resume recording again once the user is finished with the observation. The recording status should continue until the user chooses to stop the 


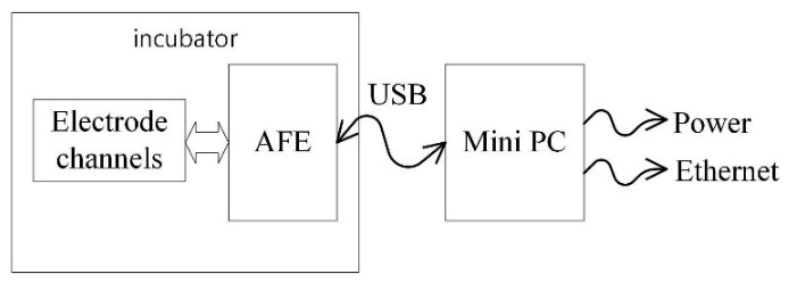

Fig. 3. Overall diagram of the proposed system.

experiment or arrive at the deadline. Since the interval of user interaction is more than several hours, it is desirable that the embedded computer, which is responsible for measuring and recording the results, occupies a small space and is protected from the inappropriate interaction of the user.

It is also advantageous not to have easy-to-access input devices such as a keyboard, a mouse, and a touch pad, or a display that occupies a large amount of space. Therefore, the system for measurement and recording is configured to be controlled through a web interface as shown in Fig. 4. This system has three states, 'idle', 'recording', and 'pause', and the four commands of 'recording', 'pause', 'resume', and 'finish' are received through a web interface. The control of transitioning from one state to another is illustrated in Fig. 4. In the recording state, the impedance based on the frequency is measured and recorded in the file system. The web interface consists of two pages: a control panel for AFE control and a report panel for presenting the impedance changes of the electrode channels.

The Analog Discovery in the AFE block was controlled using a software module written with Python using the company provided software development kit. Jupyter Notebook was used as the webserver, and communication with the AFE control software was achieved through a web socket interface. The measured impedance data were stored as text files in the mini PC. The Jupyter Notebook server was also installed on the mini PC so that web access from other computers was possible without software packages associated with Python or Jupyter Notebook. The entire software architecture is shown in Fig. 5.

The main procedure of the web server is 'measureImpedance' shown below. This procedure returns the impedance array measured for the set of channels ('channels') and the set of frequencies ('freqs'). The procedure first selects the channel in the multiplexer of AFE using the 'setMultiplexer' function for each channel. Next, it sets the frequency of the oscillator's function generator using the 'setFrequency' and 'getActualFrequency' functions for each frequency and obtain the actual set frequency. Note that the frequency of the function generator is usually set to the nearest predefined frequency. The excitation sinewave generated from the function generator and the divided voltage are sampled using the 'beginAcquisition' and 'waitAcquisitionComplete' functions. The 'get2ChannelData' function fetches the sampled sinewave data from the oscilloscope. The amplitudes and phases of excitation and the divided voltage are calculated by fitting from the data using the 'sineFit' function. From them, the complex impedance is calculated using the 'calculateGainPhase' function and 'polar2rect', and placed at the corresponding position in the output array. 


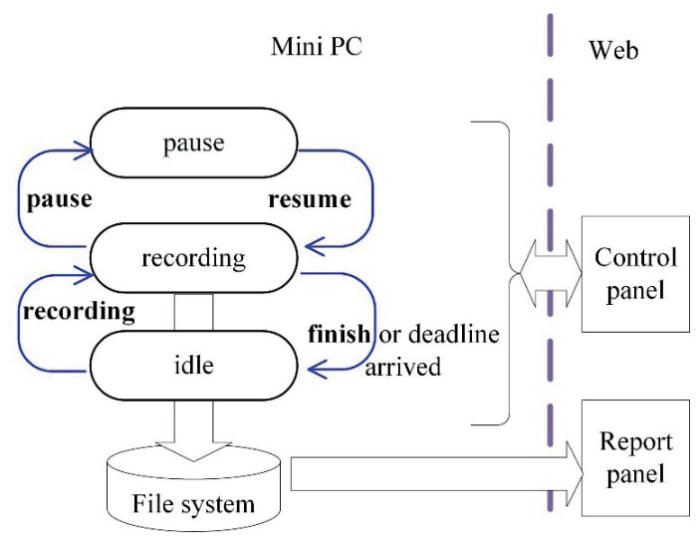

Fig. 4. (Color online) AFE control diagram.

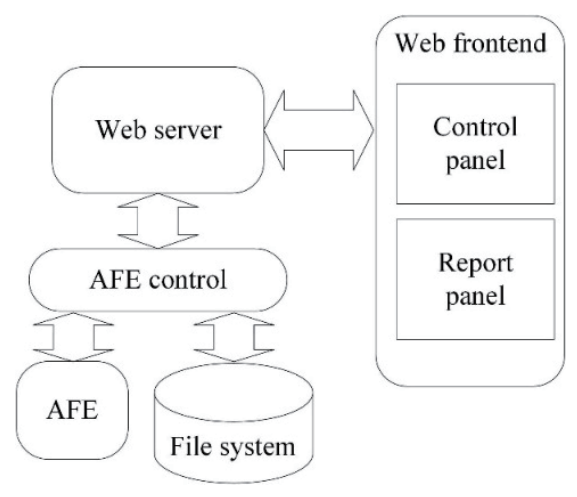

Fig. 5. Software architecture.

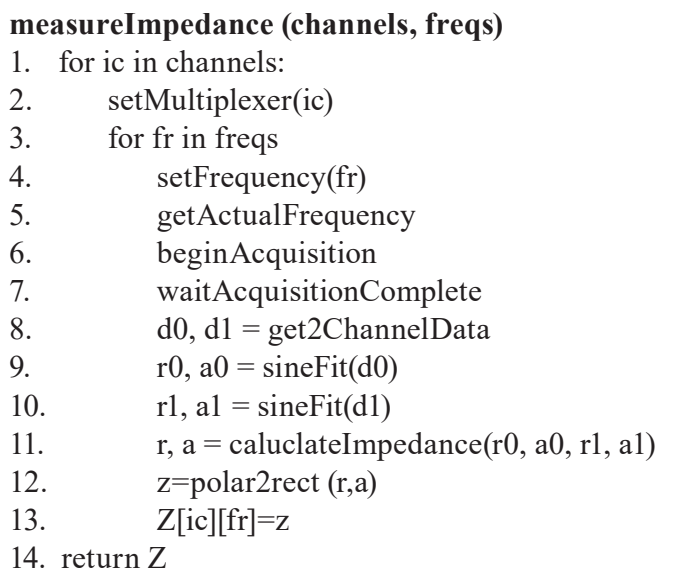

The layouts of the control and report panel are shown in Figs. 6 and 7, respectively. The 'Check' button of the control panel is used to determine whether each electrode channel is valid.

When the cell is loaded into the electrode channel, it shows a resistance of several kilo ohms, which means that there is no cell in the channel exhibiting a high resistance. When the electrode array is first installed in the system, valid channels can be detected by clicking the 'Check' button. In the list box above the 'Check' button, the measured resistance (column R) and capacitance (Column C) of each channel are displayed. Note that each channel is modeled in series of resistors and capacitances. ${ }^{(7)}$

The valid and invalid channels are indicated by the blue and red LED icons, respectively, in the upper middle part of the control panel. The check boxes next to the LED icons are used by the user to select the channel that is to be measured and recorded. The input boxes labeled as 'freqs', 'period', and 'deadline' on the upper right part of the control panel accept the required frequencies in $\mathrm{kHz}$, the measuring interval in minutes, and the experiment time in hours, respectively. When all the measurement requirements are ready, The 'Set Up' button is utilized to transmit the values to the AFE control software module. The measurement requirements received by the AFE control module can be identified throughout the whole process by pressing the 'info' button in the lower part of the control panel. The buttons labeled as 'recording', 'pause', 


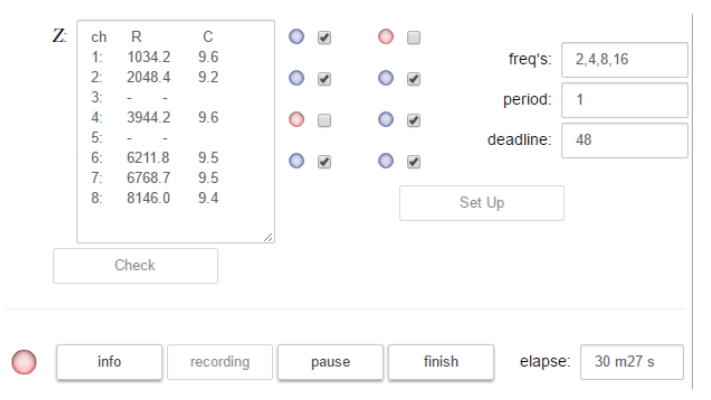

Fig. 6. (Color online) Layout of the control panel.
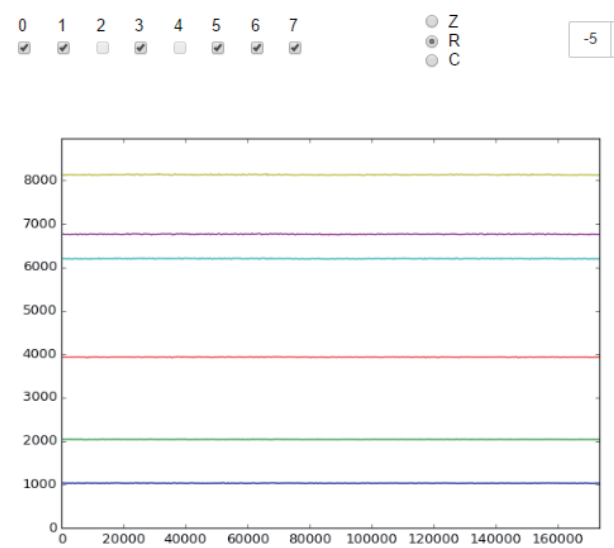

Fig. 7. (Color online) Layout of the report panel and the impedance plot of the channel emulator.

and 'finish' instruct the AFE control module to start, pause, and finish the measure/record action, respectively. The control panel is password-protected to allow access only to the main experimenter.

The module continuously records the measured data in a text file during recording. The name of the text file contains the recording start time for later analysis. The text box labeled as 'elapse' displays the time recorded so far.

The impedance change is displayed in the plot in the report channel (Fig. 7). The channels to be displayed can be selected using the check buttons in the upper part of the panel. The impedance $(\mathrm{Z})$, resistance $(\mathrm{R})$, and capacitance $(\mathrm{C})$ can be chosen using three radio buttons located in the upper middle part. The impedance change of the current or previous experiment can be chosen in the dropdown combo box located in the upper left part of the panel. The experiments are numbered in descending order, starting from the current experiment numbered as ' 0 ', the immediate previous as ' -1 ', and so on. The $x$ - and $y$-axes indicate the time in minutes from the start of the experiment and the resistance in ohms, respectively.

A test array was made to investigate the performance and usability of the proposed system (Fig. 8). Each of the six channels of the test array was placed with a series of one resistor between 1 and $8 \mathrm{k} \Omega$, and a $10 \mathrm{nF}$ capacitance to represent various impedances according to cell culture progress. Two channels (channels 3 and 5) were left open to emulate the cell-free channels.

\section{Results}

Figure 9 shows an experimental setup for measuring a test array. Only the assembly located to the right of the center vertical line is placed in the incubator, where the test array is connected to an interface board with an analog multiplexer and an amplifier using a clip, and the interface board is connected to a USB oscilloscope.

The measured impedances of the test array are shown in the list box in the upper left of the control panel (Fig. 6). The impedances of the open channels 3 and 5 in the test array were marked as '-', indicating that they exceeded the cell-free threshold. The measured resistance 


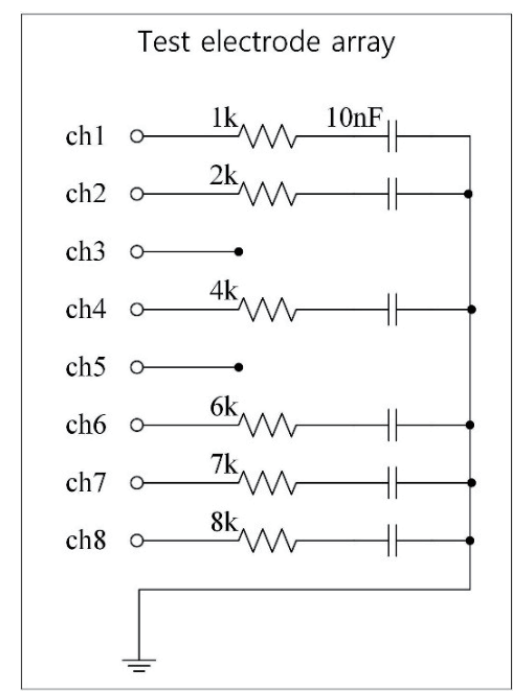

Fig. 8. Test electrode array.

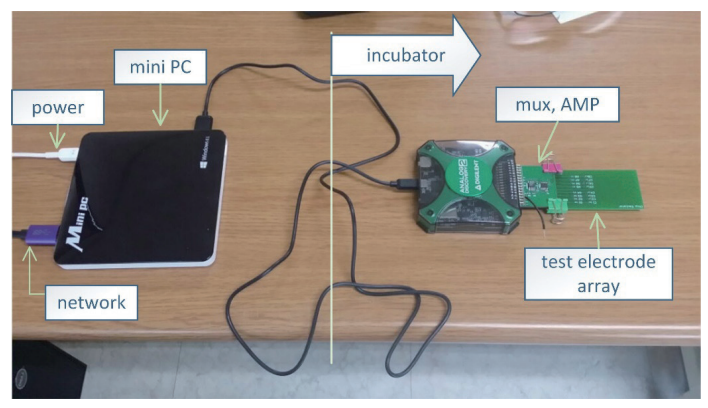

Fig. 9. (Color online) Test setup with the test electrode array.

of each channel was close to the label code of the corresponding resistor, and the capacitances were slightly less than $10 \mathrm{nF}$. Figure 7 shows the impedance change of the test array measured for more than 10 days. The graph shows that the impedance of six channels is measured with high stability. Cell-free channel detection or impedance measurement was performed stably for several months.

The results of the usability test that lasted for several months showed that there was no erroneous operation due to the carelessness of the user. When the measurement results were viewed from the outside of the laboratory, the control response or the graph drawing in the report panel was delayed sometimes due to network traffic. However, since the AFE control module continuously recorded the data, no data were lost. In addition, advantages such as sharing and discussing results with a remote user owing to the nature of the web-based interface also enhanced the usability.

In conventional systems, only array stations, which provide electrical contact to the array and having a selection circuit for each well, can be placed in the incubator. A lock-in detection system for measuring impedances, whose size is comparable to the desktop PC, should be placed outside the incubator. Because of this, a relatively large number of wires must exit the incubator, so the specially designed cable is used to connect the inside and outside of the incubator. The desk-top PC should also be positioned next to it to control the lock-in detection system and observe the measured impedance changes. Conversely, since the AFE of the proposed system, which is similar in size to the array station, includes a USB oscilloscope, only the USB signal line (4-wire) needs to be connected to the incubator. Also, since only the mini PC is needed outside the incubator, the proposed system has a large advantage in terms of size and installation compared with the existing system.

The system was provided to bioengineers to measure the well impedance of the 8 -well arrays (Standard 8 Well Arrays, Applied Biophysics, NY, USA) among commercially available culture 


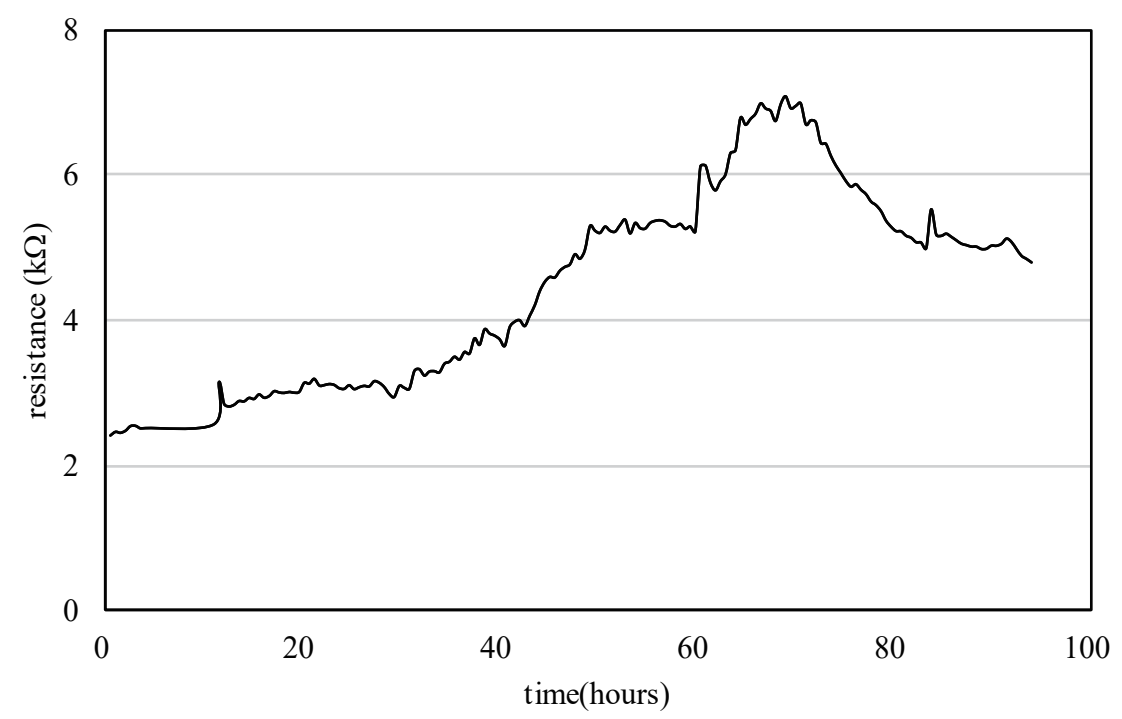

Fig. 10. Example of impedance change of a well in a cell culture experiment.

wares. Figure 10 shows an example of the impedance change measured by the proposed system, which shows a typical cell culture experiment. Impedance increases with the number of cells over time, but impedance tends to decrease again when the number of cells becomes very large.

The bioengineers using the proposed system are very satisfied with the convenient installation and the ability to monitor and discuss the experimental situation either alone or with colleagues anytime and anywhere. Data loss through careless input has not yet been reported.

To prevent the unexpected stopping of a long-running experiment, it is important to determine the status of the mini PC before the experiment by connecting a remote desktop. For example, the Windows update of the mini PC should be disabled.

When the network setup is required or a system failure occurs, $\mathrm{I} / \mathrm{O}$ devices might be connected directly to resolve the issue. In such a case, a portable monitor and a keyboard/ mouse can be employed temporarily for convenience.

\section{Conclusions}

In this study, we proposed a CPS-type ECIS system. The miniaturization of the existing system, the connection to the Internet, and the control and monitoring by the web-based graphical user interface enabled the system to escape the conventional desktop form. Because the system size was greatly reduced and there was no need for I/O devices such as displays, keyboards, and mice, the proposed system could save lab space and prevent accidental input from nearby input devices. The proposed system is being used in cell culture experiments by bioengineers and they are very satisfied with the ease of installation and ubiquitous observation of the experimental situation and discussions with colleagues. The user interface and sensing functions of the proposed system are separated by the server and web client, so they can easily migrate to a cloud-connected system in the future. 


\section{Acknowledgments}

This research was supported by the Basic Science Research Program through the National Research Foundation of Korea (NRF) funded by the Ministry of Science, ICT \& Future Planning (NRF-2017R1A2B4002679), and by The Leading Human Resource Training Program of Regional Neo Industry through the National Research Foundation of Korea (NRF) funded by the Ministry of Science, ICT \& Future Planning (NRF-2016H1D5A1909654).

\section{References}

1 R. C. Nordberg, J. Zhang, E. H. Griffith, M. W. Frank, B. Starly, and E. G. Loboa: Stem Cells Transl. Med. 6 (2017) 502.

2 T. Schwarzenberger, P. Wolf, M. Brischwein, R. Kleinhans, F. Demmel, A. Lechner, B. Becker, and B. Wolf: Physiol. Meas. 32 (2011) 977.

3 Y. Zhou, S. Basu, E. Laue, and A. A. Seshia: Biosens. Bioelectron. 81 (2016) 249.

4 F. Liu, F. Li, A. N. Nordin, and I. Voiculescu: Sensors 13 (2013) 3039.

5 C. Xiao and J. H. Luong: Toxicol. Appl. Pharmacol. 206 (2005) 102.

6 J. Wegener, C. R. Keese, and I. Giaever: Exp. Cell. Res. 259 (2000) 158.

7 I. Giaever and C. R. Keese: Proc. Natl. Acad. Sci. 88 (1991) 7896.

8 J. Gubbi, R. Buyya, S. Marusic, and M. Palaniswami: Future Gener. Comput. Syst. 29 (2013) 1645.

9 A. F. A. Rahman, M. Daud, and M. Z. Mohamad: Proc. Int. Conf. Internet of things and Cloud Computing (2016) 79.

10 A. Botta, W. De Donato, V. Persico, and A. Pescapé: Future Gener. Comput. Syst. 56 (2016) 684.

11 W. Huang, F. Fang, X. Ma, Z. Dong, and X. Xu: 2015 IEEE 10th Conf. Industrial Electronics and Applications (ICIEA, 2015) 2030.

12 M. Qi, G. Cheng, Y.-1. Xu, K. Li, C. Gong, X. Yue-Lei, and L. Ke: 2014 Int. Conf. Automatic Control Theory and Application (ACTA-14, 2014).

13 P. K. Bhagwat and H. Zodpe: 2014 Int. Conf. Embedded Systems (ICES, 2014) 157.

14 S. B. Khot and M. S. Gaikwad: 2016 Int. Conf. Computing Communication Control and automation (ICCUBEA, 2016) 1.

15 G. Mashette, P. Borole, and S. Bhat: 2016 IEEE Int. Conf. Recent Trends Electronics, Information \& Communication Technology (RTEICT, 2016) 1407.

16 A. Lewis, M. Campbell, and P. Stavroulakis: Measurement 87 (2016) 228.

17 A. Ukil, S. Bandyoapdhyay, C. Puri, and A. Pal: 30th IEEE Int. Conf. Advanced Information Networking and Applications (AINA, 2016) 994. 